\title{
Safety, Effectiveness, and Treatment Persistence of Golimumab in Elderly Patients with Rheumatoid Arthritis in Real-World Clinical Practice in Japan
}

\author{
Masateru Okazaki (D) Hisanori Kobayashi - Hirohito Shimizu • \\ Yutaka Ishii · Tsutomu Yajima • Masayoshi Kanbori
}

Received: January 16, 2018 / Published online: March 2, 2018

(C) The Author(s) 2018. This article is an open access publication

\begin{abstract}
Introduction: Golimumab has been proven as an effective treatment for rheumatoid arthritis in clinical trials. However, there is a scarcity of data regarding its use in elderly patients in a real-world setting. This study aims to evaluate the safety, effectiveness, and treatment persistence of golimumab in elderly Japanese patients ( $\geq 75$ years) with rheumatoid arthritis.

Methods: This study was a post hoc analysis of post-marketing surveillance data on 5137 Japanese patients with active rheumatoid arthritis
\end{abstract}

Enhanced content To view enhanced content for this article go to https://doi.org/10.6084/m9.figshare.5900 914.

Electronic supplementary material The online version of this article (https://doi.org/10.1007/s40744018-0101-y) contains supplementary material, which is available to authorized users.

M. Okazaki $(\varangle) \cdot$ H. Kobayashi · H. Shimizu · Y. Ishii Immunology Department, Medical Affairs Division, Janssen Pharmaceutical K.K., Tokyo, Japan e-mail: mokazak1@its.jnj.com

\section{T. Yajima}

Biostatistics Department, Research and

Development Division, Janssen Pharmaceutical

K.K., Tokyo, Japan

\section{Kanbori}

Japan Safety and Surveillance Division, Research and Development Division, Janssen Pharmaceutical K.K., Tokyo, Japan who received golimumab for 24 weeks. The study population was divided into two age groups (younger: < 75 years and elderly: $\geq 75$ years), and the safety, effectiveness, and treatment persistence of golimumab were assessed. Also, the reasons for discontinuing golimumab treatment were analyzed by multilogistic regression.

Results: During golimumab treatment over 24 weeks, younger and elderly groups exhibited comparable improvement of disease activity as measured by EULAR response criteria with similar overall rates of adverse events. However, the survival curve of golimumab for elderly patients was significantly different from that for younger patients due largely to the discontinuation at 4 weeks. The most common reason for discontinuation in elderly patients was patient choice, while it was disease progression in younger patients. Analysis of elderly patients who discontinued treatment by their own decision identified EULAR good response as a factor associated with continuation of golimumab treatment whereas no predictive factor associated with discontinuation was identified. Conclusions: The safety and effectiveness of golimumab treatment in elderly Japanese patients aged 75 years or older were comparable to those in younger patients in real-world clinical practice. Analysis of the survival curves suggested that continuous use of golimumab might further improve clinical benefit of golimumab in elderly patients, underpinning the 
importance of effective communication between physicians and elderly patients based on the treat-to-target strategy.

Funding: Janssen Pharmaceutical K.K. and Mitsubishi Tanabe Pharma Corporation.

Keywords: Elderly patient; Effectiveness; Golimumab; Post-marketing surveillance; Realworld evidence; Rheumatoid arthritis; Safety; Treatment persistence

\section{INTRODUCTION}

Rheumatoid arthritis (RA) is a highly prevalent autoimmune disease, hallmark of which is chronic inflammation and progressive destruction of multiple joints [1]. Patients with RA are at significantly increased risk of various comorbidities and mortality compared to the general population [2-4]. The prevalence of RA in developed countries is reportedly more than double the global estimate: $0.53-0.55 \%$ for the US [5], $0.67 \%$ for the UK [6], and $0.6-1.0 \%$ for Japan [7], as opposed to $0.24 \%$ for the global population [8]. A recent systematic review suggested that the global burden of RA was increased by approximately 45\% from 1990 to 2010 due to accelerated population growth combined with continued population aging [8]. Indeed, it has been suggested that the peak age at onset of RA has increased from 50-59 years in 2002-2003 to 60-69 years in 2012-2013 in Japan [9], necessitating better understanding of elderly RA in a real-world clinical setting and development of its treatment strategies. In recent years, several biologic disease-modifying antirheumatic drug (bDMARD) options for RA have become available targeting mediators of inflammation process such as tumor necrosis factor alpha $(\mathrm{TNF} \alpha)$ and other cytokines [10]. Accumulating evidence indicates that biologic treatments result in reduced progression of joint damage, rapid remission, and improved quality of life $[11,12]$. The introduction of golimumab (GLM) in 2011 provided Japanese patients with RA an opportunity for a new treatment option. GLM is a human monoclonal antibody developed to inhibit signaling activities of $\mathrm{TNF} \alpha$ $[13,14]$ and indicated for the treatment of several rheumatic diseases such as active RA [15], ankylosing spondylitis [16], and psoriatic arthritis [17]. In Japan, GLM is approved to be administered subcutaneously for the treatment of moderate to severe RA inadequately responded to conventional therapies.

Recent clinical studies in Japan, GO-FORTH [18] and GO-MONO [19], have demonstrated efficacy and safety of GLM in patients with active RA. However, elderly patients aged 75 years or over were excluded in both GLM clinical trials despite the fact that patients aged 70 years or over account for $35.4 \%$ of the total number of patients enrolled in the post-marketing surveillance (PMS) of GLM [20]. The elderly population is therefore underrepresented in these clinical trials, thereby hampering the generalizability of the efficacy and safety to the real-world patient population.

Treatment persistence, which is defined as a duration of time from initiation to discontinuation of treatment, has been suggested as a measurable factor that can serve as a proxy for long-term therapeutic benefit and harm in the real world [21]. Indeed, this notion is supported by previous studies demonstrating that poor treatment persistence of biologics can blunt the effectiveness $[22,23]$. While several studies have revealed that overall treatment persistence of biologic agents in elderly RA patients is not inferior to that in younger RA patients [24, 25], large variation in treatment persistence as well as reasons for discontinuation among different biologic agents have been noted $[25,26]$. As to GLM, there are no published studies on treatment persistence measured for patients aged 75 years or above in real-world practice in Japan.

We undertook a post hoc analysis of a PMS study that included 5137 Japanese RA patients who were treated with GLM for 24 weeks. The primary objective of this study was to compare the safety and effectiveness of GLM between elderly ( $\geq 75$ years) and younger ( $<75$ years) patient populations. We also assessed treatment persistence of GLM together with the reasons for discontinuing GLM in these populations to examine whether there is any trend characteristic to elderly RA patients aged 75 years or older. 


\section{METHODS}

\section{Data Source and Study Design}

The present study was a post hoc retrospective analysis of a post-marketing surveillance (PMS) study (UMIN000015895) that included 5137 Japanese patients with RA who were centrally registered and treated with GLM between September 2011 and May 2013. The PMS was an open-label, non-interventional, observational study that was conducted on patients with active RA at selected medical facilities across Japan during the 24-week surveillance period. The protocol and ethical considerations for the PMS were assessed by the internal review board members and approved by the Japanese Pharmaceutical Medical Device Agency (PMDA). This PMS was conducted under the Japanese regulations (Ministry of Health, Labor and Welfare Ministerial Ordinance No. 171) for Good Post-Marketing Study Practice (GPSP), and was formally initiated following signing of an agreement with each study center.

\section{Participants and Data Collection}

In this post hoc analysis, data of 5137 RA patients registered in the PMS were classified according to the age at the time:

1. the younger group consisting of patients under 75 years of age ( $<75$ years),

2 . the elderly group consisting of patients aged 75 years or over ( $\geq 75$ years).

Data including demographics (age, gender, body weight, and disease duration) as well as clinical characteristics (such as disease activity score in 28-joints [DAS28], current and previous treatments for RA, and associated comorbidities) for both patient groups were obtained.

\section{Statistical Analysis}

Categorical variables were displayed as frequencies and proportions (\%), while continuous variables were presented as the mean and standard deviation (SD) unless otherwise described. To compare unpaired continuous variables, Student's $t$ test or Welch's $t$ test was performed. For comparison of categorical data, Chi-squared test was employed. Treatment persistence for both the elderly group (age $\geq 75$ ) and younger group $($ age $<75)$ is shown by Kaplan-Meier curves and the difference was analyzed using log-rank test. All statistical analyses were completed using R Software Version 3.2.5 [27] or SAS version 9.3 (SAS Institute Inc., Cary, NC, USA) without imputation of missing data. A two-sided $p$ value less than 0.05 was considered statistically significant.

\section{Analysis of the Reasons for Discontinuing GLM Treatment}

When discontinuing GLM treatment, participants were prompted with a list of issues (patient choice, disease progression, comorbidities, adverse event (AE), death, hospital transfer, noshow, other reasons) from which to select the one best categorizes the reason for discontinuation. Note that "discontinuation" is defined as stopping GLM treatment due to an unavoidable reason, which excludes the case when patients stopped GLM treatment due to significant improvement of disease activity. It should also be noted that "disease progression", "comorbidities", and "AE" were all medical decisions that were made by physicians, whereas "patient choice" was a non-medical decision solely initiated by patients independent of physicians' judgement. Reasons for the discontinuation of GLM treatment during the study were analyzed in each of the younger and the elderly groups and presented as bar graphs. We then analyzed demographic and clinical characteristics of the elderly patients in order to identify predictive factors associated with discontinuation of GLM treatment due to patient choice. For each of the younger and elderly groups, the demographic and clinical profiles of the patients who continued GLM treatment were compared to those who discontinued treatment due to patient choice. Other reasons such as disease progression and $\mathrm{AE}$ were not included in this analysis.

Univariate and multivariate logistic regression analyses were performed to identify patient characteristic variables (e.g., demographic data, current treatment, concomitant medication, 
and AEs) associated with discontinuation of GLM due to patient choice. Univariate logistic regression analysis was performed for the patient demographic factors with $p<0.10$ in other analyses, and then multivariate analysis was subsequently conducted by using variables with $p<0.20$ in the univariate analysis. The results are presented as odds ratios (ORs) with 95\% confidence intervals (CIs).

\section{RESULTS}

\section{Study Population and Baseline Characteristics}

The baseline demographics together with the baseline characteristics of the patients are summarized in Table 1. Of the total RA patients examined, $82 \% \quad(n=4200)$ were categorized into the younger group and $18 \%(n=937)$ into the elderly group. The mean ages for the younger and the elderly patients were $59.0 \pm 11.8$ and $79.0 \pm 3.7$ years, respectively. The elderly group had similar males to the younger group ( 19.0 vs. $18.4 \%, p=0.695)$. The elderly group exhibited higher disease activity than younger patients as indicated by DAS28ESR $(4.9 \pm 1.4$ vs. $4.7 \pm 1.4, p<0.001)$, DAS28CRP $(4.2 \pm 1.3$ vs. $4.0 \pm 1.3, p=0.005)$, SDAI $(22.9 \pm 13.5$ vs. $21.3 \pm 12.7, p=0.006)$ and CDAI $(20.8 \pm 12.6$ vs. $19.6 \pm 11.9$, $p=0.037)$ while the differences in tender joint count (TJC) $(5.2 \pm 5.7$ vs. $5.2 \pm 5.5, p=0.882)$ and swollen joint count (SJC) $(4.9 \pm 5.1$ vs. $4.7 \pm 4.6, p=0.374$ ) were not statistically significant. The mean number of previous biologic DMARDs was significantly higher in the younger group than in the elderly group $(0.9 \pm 1.0$ vs. $0.8 \pm 0.9, p=0.044)$. Overall, elderly patients suffer from comorbidities more frequently than younger patients. Chronic comorbid conditions such as hypertension (30.8 vs. $16.1 \%, p<0.001$ ) and diabetes mellitus (13 vs. $7.9 \%, p<0.001)$ were more prevalent among elderly patients. Other comorbidities that were more frequently observed in the elderly group included respiratory disease (7.9 vs. $5 \%, p<0.001)$ and abnormal renal function (11.2 vs. $3.9 \%, p<0.001)$.

\section{Safety and effectiveness of GLM in Elderly and Younger RA Patients}

The use of concomitant medications is summarized in Table 2. Despite comparable GLM dosage $(60.5 \pm 18.8$ for younger vs. $61.1 \pm 19.4$ for elderly, $p=0.395$ ), elderly patients received methotrexate (MTX) as a concomitant medication at a lower dosage $(6.3 \pm 2.6$ vs. $8.2 \pm 3.1$, $p<0.001)$ and less frequently (67.9 vs. $82.4 \%$, $p<0.001)$ than younger patients. In contrast, elderly patients used csDMARDs other than MTX more frequently than younger patients (33.5 vs. $28.2 \%, p=0.001$ ). Glucocorticoid (GU) was concomitantly administered to elderly patients more frequently $(56.0$ vs. $51.2 \%$, $p=0.008)$ but at a lower dosage $(4.3 \pm 3.0$ vs. $4.6 \pm 2.7, p<0.033)$ than younger patients.

There was no significant difference in the overall incidence of AEs between the two populations in this study $(21.4 \%$ for younger vs. $19.1 \%$ for elderly, $p=0.137)$. We noted that infectious diseases, abnormal liver function, and hematologic disorders were the most common events both in younger and elderly patients. When each AE type was compared between the two groups, the incidence rate of cardiac disorder was higher in elderly patients than younger patients ( 1.0 vs. $0.3 \%, p=0.013)$, while that of abnormal liver function was higher in younger patients (5.3 vs. $1.5 \%$, $p<0.001)$. Regarding the effectiveness, both groups demonstrated comparative improvement of disease activity as measured by EULAR response criteria ( $p=0.837$, Table 2$)$ although overall clinical characteristics of RA at baseline were worse in the elderly group than in the younger group (Table 1).

\section{Treatment Persistence of GLM in Elderly and Younger Patients}

To evaluate the long-term therapeutic benefit and risk of GLM in the real world, we analyzed treatment persistence of GLM, which is measured as the time between first administration to discontinuation. Discontinuation in this context refers to stopping GLM treatment due to an unavoidable reason, excluding the case in 
Table 1 Baseline demographic data and clinical characteristics in younger (age $<75)$ and elderly patients (age $\geq 75$ )

\begin{tabular}{lllr}
\hline Patient characteristics & $\begin{array}{l}\text { Younger }(\mathbf{a g e}<\mathbf{7 5}) \\
\boldsymbol{n}=\mathbf{4 2 0 0}\end{array}$ & $\begin{array}{l}\text { Elderly }(\text { age } \geq \mathbf{7 5}) \\
\boldsymbol{n}=\mathbf{9 3 7}\end{array}$ & $\boldsymbol{p}$ value \\
\hline Age (years) & $59.0 \pm 11.8$ & $79.0 \pm 3.7$ & $<0.001$ \\
Male (\%) & $772(18.4)$ & $178(19.0)$ & 0.695 \\
Body weight (kg) & $54.7 \pm 10.9$ & $49.9 \pm 9.9$ & $<0.001$ \\
Disease duration (years) & $10.8 \pm 10.0$ & $11.9 \pm 11.4$ & 0.013 \\
ESR (mm/h) & $39.5 \pm 28.8$ & $48.1 \pm 30.8$ & $<0.001$ \\
CRP (mg/dl) & $1.6 \pm 2.2$ & $2.0 \pm 2.6$ & $<0.001$ \\
DAS28ESR & $4.7 \pm 1.4$ & $4.9 \pm 1.4$ & $<0.001$ \\
DAS28CRP & $4.0 \pm 1.3$ & $4.2 \pm 1.3$ & 0.005
\end{tabular}

Steinbrocker's stage

I/II/III/IV (\%)

Steinbrocker's class

$$
\text { I/II/III/IV (\%) }
$$

TJC

SJC

Patient's VAS

Physician's VAS

SDAI

CDAI

Number of previous use of biologics

Comorbidities

$\begin{array}{ll}\text { Abnormal renal function (\%) } & 162(3.9) \\ \text { Abnormal liver function (\%) } & 479(11.4) \\ \text { Respiratory disease (\%) } & 211(5.0) \\ \text { Diabetes mellitus (\%) } & 331(7.9) \\ \text { Hypertension (\%) } & 675(16.1) \\ \text { Hyperlipidemia (\%) } & 364(8.7)\end{array}$

$5.2 \pm 5.5$

$4.7 \pm 4.6$

$0.9 \pm 1.0$

$$
\begin{aligned}
& \text { 601/1219/1109/1146 (14.3/29.0/26.4/ 77/273/293/273 (8.2/29.1/31.3/ }<0.001 \\
& \text { 27.3) } \\
& \text { 29.1) }
\end{aligned}
$$

$716 / 2445 / 827 / 89(17.0 / 58.2 / 19.7 / 2.1)$

$63 / 528 / 277 / 49(6.7 / 56.4 / 29.6 /<0.001$ 5.2)

$5.2 \pm 5.7$

0.882

$4.9 \pm 5.1$

0.374

$49.1 \pm 25.8$

$53.1 \pm 25.7$

$<0.001$

$44.9 \pm 23.5$

$47.8 \pm 23.7$

0.005

$21.3 \pm 12.7$

$22.9 \pm 13.5$

0.006

$19.6 \pm 11.9$

$20.8 \pm 12.6$

0.037

$0.8 \pm 0.9$

0.044

105 (11.2) $<0.001$

$93(9.9)$

0.213

$74(7.9)$

$<0.001$

$122(13.0)$

$<0.001$

$289(30.8)$

$<0.001$

$106(11.3)$
0.013

Data reported as mean \pm standard deviation unless otherwise stated

CDAI clinical disease activity index, CRP C-reactive protein, DAS28ESR/CRP disease activity score in 28-joints based on ESR/CRP, ESR erythrocyte sedimentation rate, Patient's VAS patient's overall assessment on a VAS, Physician's VAS physician's overall assessment on a VAS, SDAI simplified disease activity index, SJC swollen joint count, TJC tender joint count, $V A S$ visual analogue scale 
Table 2 Treatment characteristics, adverse events, and effectiveness of GLM treatment in younger (age $<75$ ) and elderly patients (age $\geq 75$ )

$\begin{array}{ll}\begin{array}{l}\text { Younger }(\text { age }<75) \\ n=4200\end{array} & \begin{array}{l}\text { Elderly (age } \geq 75) \\ n=937\end{array}\end{array}$

GLM and concomitant therapy

GLM dose (mg/time)

$60.5 \pm 18.8$

$61.1 \pm 19.4$

0.395

Concomitant medication use

MTX (\%)

$3459(82.4)$

$636(67.9)$

$<0.001$

csDMARDs (other than MTX) (\%)

$1183(28.2)$

$314(33.5)$

0.001

GCs (\%)

$2149(51.2)$

$525(56.0)$

0.008

Dose of concomitant medication

$$
\begin{aligned}
& \text { MTX (mg/week) } \\
& \text { GC (mg/day) }
\end{aligned}
$$

Total number of patients with AEs (\%)

Malignancy (\%)

Infectious diseases (\%)

Serious allergic reaction (\%)

Interstitial lung disease (\%)

Autoimmune disease (\%)

Demyelinating disease (\%)

Cardiac disorder (\%)

Hematologic disorders (\%)

Abnormal liver function (\%)

EULAR response criteria ${ }^{a}$

No response (\%)

Moderate response (\%)

Good response (\%)

$\begin{array}{lll}8.2 \pm 3.1 & 6.3 \pm 2.6 & <0.001 \\ 4.6 \pm 2.7 & 4.3 \pm 3.0 & 0.033 \\ 897(21.4) & 179(19.1) & 0.137 \\ 19(0.5) & 6(0.6) & 0.626 \\ 310(7.4) & 65(6.9) & 0.687 \\ 5(0.1) & 0(0.0) & 0.633 \\ 25(0.6) & 6(0.6) & 1.000 \\ 19(0.5) & 5(0.5) & 0.948 \\ 0(0.0) & 0(0.0) & \mathrm{NA} \\ 13(0.3) & 9(1.0) & 0.013 \\ 69(1.6) & 12(1.3) & 0.510 \\ 223(5.3) & 14(1.5) & <0.001 \\ n=2167 & n=438 & \\ 693(32.0) & 145(33.1) & 0.837 \\ 531(24.5) & 109(24.9) & \\ 943(43.5) & 184(42.0) & \end{array}$

csDMARDs conventional synthetic biological disease-modifying antirheumatic drugs, EULAR European league against rheumatism, GCs glucocorticoids, GLM golimumab, MTX methotrexate, NA not applicable

Data reported as mean \pm standard deviation unless otherwise stated

${ }^{a}$ Patients were evaluated by EULAR response criteria (DAS28CRP) following the final treatment including the patients who discontinued treatment

which patients have stopped GLM treatment because of significant improvement of disease activity. Kaplan-Meier curves for time to discontinuation of GLM for each study group are shown in Fig. 1a. The mean persistence in weeks with GLM over 24 weeks was 21.0 for the younger group and 20.3 for the elderly group. The discontinuation rate due to any reason during 24 weeks of study period was $22.0 \%$ $(n=925 / 4200)$ for the younger group whereas 
that of the elderly group was $25.3 \%(n=237 /$ 937). Consistently, the survival curves of GLM were statistically different between the two groups $(p=0.015)$ as determined by the logrank test (Fig. 1a). We noted that this discrepancy results predominantly from the relatively high discontinuation rate of the elderly group at 4 weeks (Fig. 1a). In addition, there was a difference in the frequency of discontinuation by reason between the two study groups. In the younger patient group, progression of disease was the most frequently reported reason (31.2\%), followed by patient choice $(25.8 \%)$ and AE (20.8\%) (Fig. 1b). Conversely, in the elderly patient group, the patient choice $(26.2 \%)$ was rated highest, followed by the progression of disease $(23.2 \%)$ and $\mathrm{AE}(18.1 \%)$ as the reasons for discontinuing GLM treatment (Fig. 1c).

In each of the younger and the elderly patient study populations, we compared patients who continued GLM treatment through 24 weeks with those discontinued GLM treatment due to patient choice by 24 weeks (supplementary Tables S1, S2). Univariate and multivariate regression models were employed to identify demographic and clinical factors associated with discontinuation of GLM treatment due to patient choice (Fig. 2). In the younger group, multivariate analysis identified only EULAR non-response as the factor significantly associated with discontinuation of GLM treatment by patient choice $(\mathrm{OR}=5.030,95 \%$ CI; 2.603-9.722, $p<0.001)$, suggesting that those who showed EULAR non-response were likely to continue GLM treatment. In the elderly patient groups, however, EULAR good response was solely identified as the predictor associated with continuation of GLM treatment by patient choice $\quad(\mathrm{OR}=0.225, \quad 95 \% \quad \mathrm{CI} ; \quad 0.057-0.894$, $p=0.034)$, suggesting that those who showed EULAR good response were more likely to continue GLM treatment.

\section{DISCUSSION}

With the advent of the era of a "super-aged society" [28], the population of elderly people diagnosed with RA is on the rise [8]. A database study has demonstrated that the peak age at onset of RA has shifted from 50-59 to 60-69 years of age over the past decade [9], suggesting that more elderly people tend to start suffering from RA in Japan. Clinical characteristics of the elderly population are different from those of younger patients, making it difficult to interpret the data from clinical trials, which generally exclude elderly subjects to achieve homogeneous study population. This constraint of study design compromises the extrapolation of data from clinical trials to the elderly population in a real-world clinical setting. Alternatively, there are accumulating realworld studies investigating the use of biologics to treat elderly RA patients where the chronological age of 60 or 65 years is typically used as a definition of "elderly" [29]. These cutoffs, however, may not adequately reflect the population in a real-world setting under the super-aged society, where majority of elderly people remain physically and mentally robust. In fact, a proposal has been made to redefine the elderly as aged 75 years and older in Japan [30].

Here we defined the chronological age of 75 years as a threshold for elderly to evaluate the safety and effectiveness of GLM treatment in elderly Japanese patients using data from the PMS of GLM in 5137 Japanese patients with active RA. While the safety and effectiveness of GLM in the younger group reported here corroborate previous clinical trial data $[18,19,31,32]$, the current study demonstrated, for the first time, that the safety and effectiveness of GLM in elderly (75 $\leq$ years) Japanese patients with active RA are comparable to those in younger ( $75>$ years) patients. It should be noted that, in elderly patients, the frequency of concomitant MTX use, which has been proven to be effective for anti-TNF agents, is lower than that in younger patients albeit with higher baseline disease activity. Thus, it appears that the effectiveness of GLM in elderly RA patients is not confounded by either baseline disease activity or concomitant MTX use. Moreover, our analysis suggested that elderly patients tend to discontinue GLM during early treatment phase by their own decision but those who exhibit EULAR good response are likely to continue GLM treatment. 


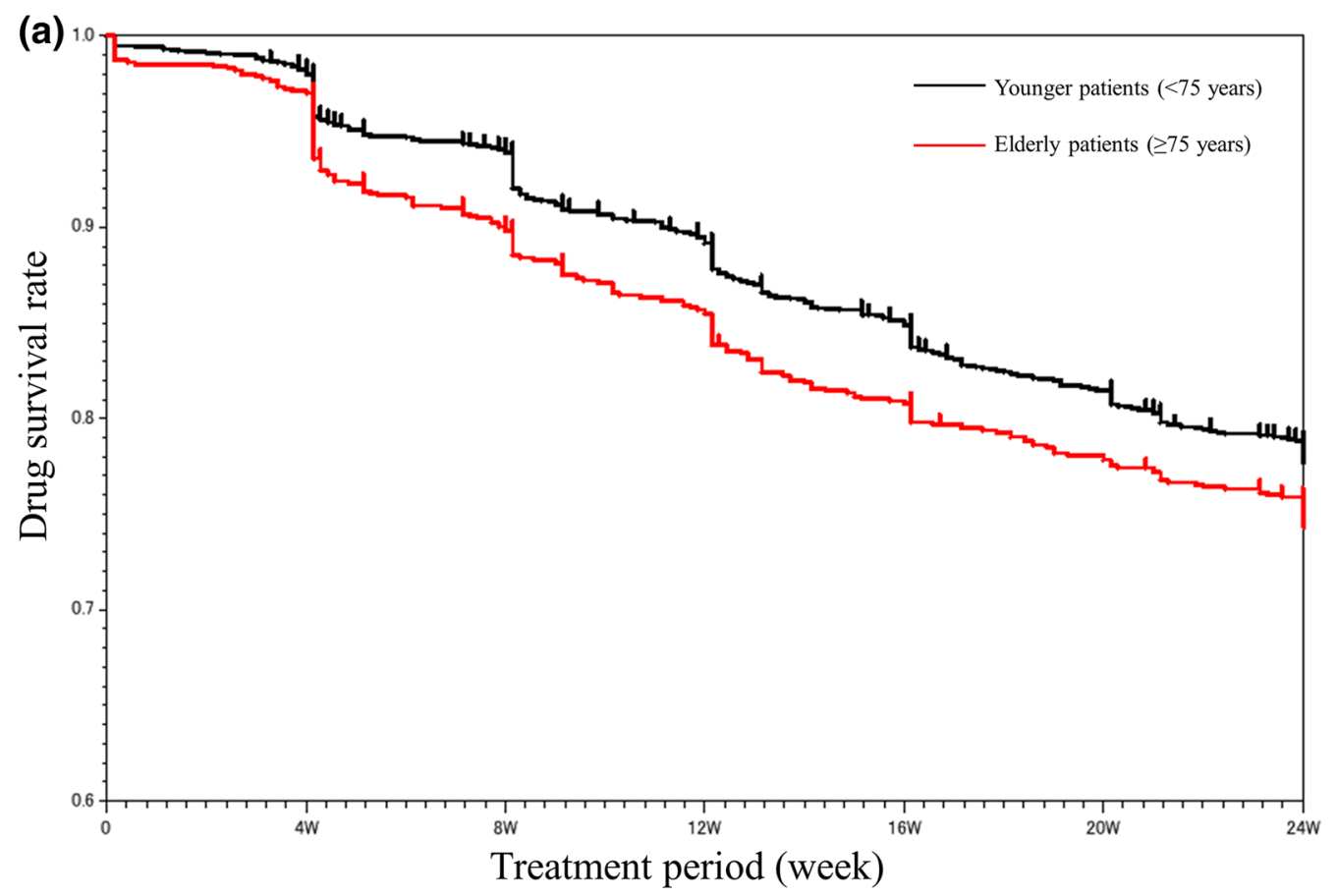

(b) Younger patients $(<75)$

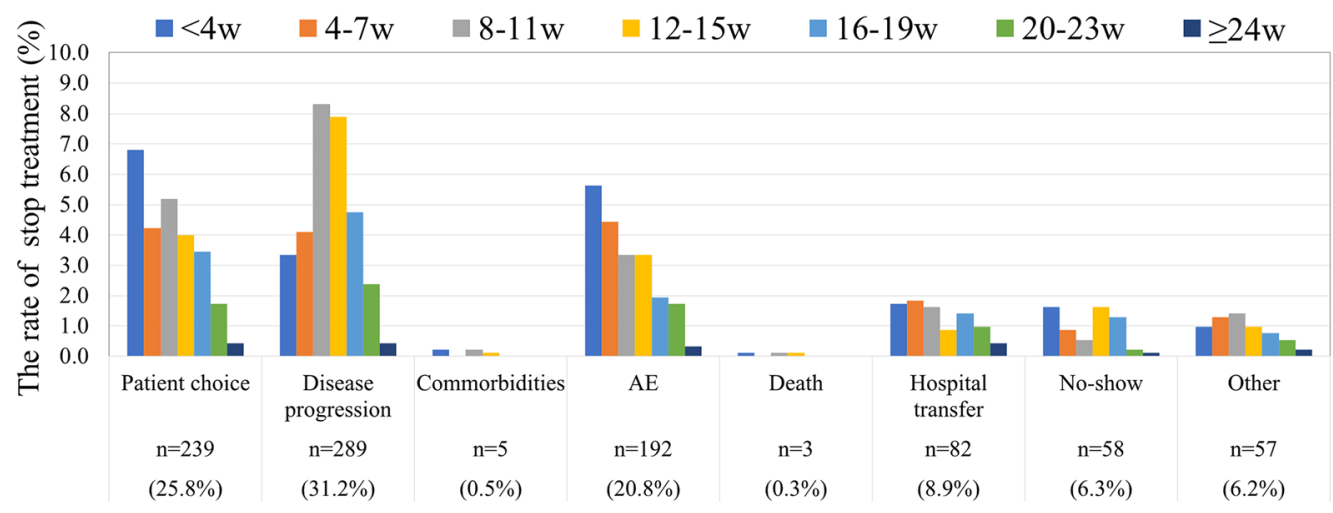

(c) Elderly patients $(\geq 75)$

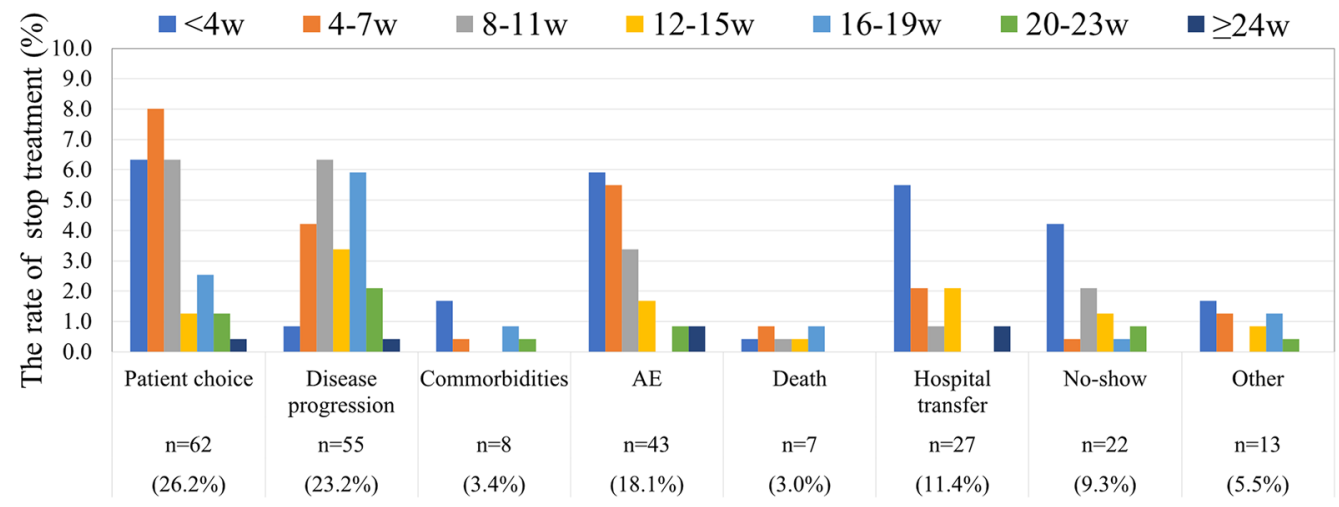


4Fig. 1 Discontinuation of GLM treatment. a Kaplan-Meier curve of time to discontinuation for GLM among younger and elderly patients. Survival curves of GLM were compared using the log-rank test between the two groups $(p=0.015)$. b Relative frequency of reasons for discontinuing GLM treatment in younger patients (age $<75$ ). c Relative frequency of reasons for discontinuing GLM treatment in elderly patients (age $\geq 75$ ). The proportion of patients citing each reason as a primary cause of discontinuation of GLM is shown in each period analyzed

Interestingly, the current study revealed that the treatment regimen for patients with RA is, indeed, notably different between younger and elderly patients. MTX is one of the most typical instances, where physicians tend to limit its use for elderly patients due probably to high incidence of comorbidities such as abnormal renal functions in this patient population. This trend might reflect real-world clinical practice in which physicians follow Japanese guidelines for the use of MTX that recommend careful monitoring of elderly patients to minimize the risk of AEs [33]. In contrast, other csDMARDs and GCs are more commonly used to treat this patient group probably because the AEs are reportedly lower than those caused by MTX [34-36]. Interestingly, our data further indicate that the elderly patient group received a similar dose of GLM to that of the younger patient group. These findings suggest that physicians in realworld clinical practice adjust the doses of csDMARDs and GCs to maximize the effectiveness of GLM while at the same time minimize the risks of AEs. As a result, GLM treatment appeared to be consistent in effectiveness between the two groups, although elderly patients received lower dose of MTX than younger patients.

Despite no difference in the overall incidence rate of AEs between the younger and elderly patients, statistically significant differences were observed in the rate of cardiac disorders and abnormal liver function between the two groups. It is speculated that the higher rate of cardiac disorders in the elderly group with RA might be associated with greater risk factors such as hypertension, hyperlipidemia, diabetes mellitus, and chronic kidney disease in this population [37-40]. In contrast, abnormal liver function was observed at a higher rate in the younger group due possibly to the increased rate of MTX use in these patients as MTX is known to induce hepatotoxicity [41].

As opposed to the safety and effectiveness profiles, the survival curve of GLM treatment for elderly patients was found to be statistically different from that for younger patients due primarily to the relatively high discontinuation rate observed at 4 weeks probably before the full clinical effect of GLM was achieved. Moreover, the primary reason for GLM discontinuation was different between the two groups. In the younger group, the progression of disease was the main reason for discontinuing GLM treatment throughout the study period, whereas, in the elderly group, the patient choice was the main reason. This result still holds true when analyzed specifically at 4 weeks, at which we identified a significant decline of the GLM continuation rate in the elderly group. Thus, our analysis revealed a characteristic patient journey for elderly patients treated with GLM; compared to younger patients, they are more likely to discontinue GLM by their own decision even before physicians are able to legitimately evaluate if patients are likely or unlikely to respond to GLM.

We then investigated putative predictive factors associated with discontinuation of GLM due to patient choice. Univariate and multivariate regression analyses solely identified EULAR non-response [10, 42] as a factor associated with discontinuation of GLM treatment by patient choice in the younger patient group, while only EULAR good response was associated with continuation of GLM treatment in the elderly patient group. This result in itself appears empirically explicit but the association between treatment satisfaction and persistence has been indeed demonstrated based on the rigorous review of literature [43]. However, it should be noted that, unlike younger patients, we failed to identify EULAR non-response as the factor associated with discontinuation of GLM treatment due to patient choice in elderly patients. Thus, an identification of the key issues in "patient choice" will be of future 
(a) Younger $(<75)$

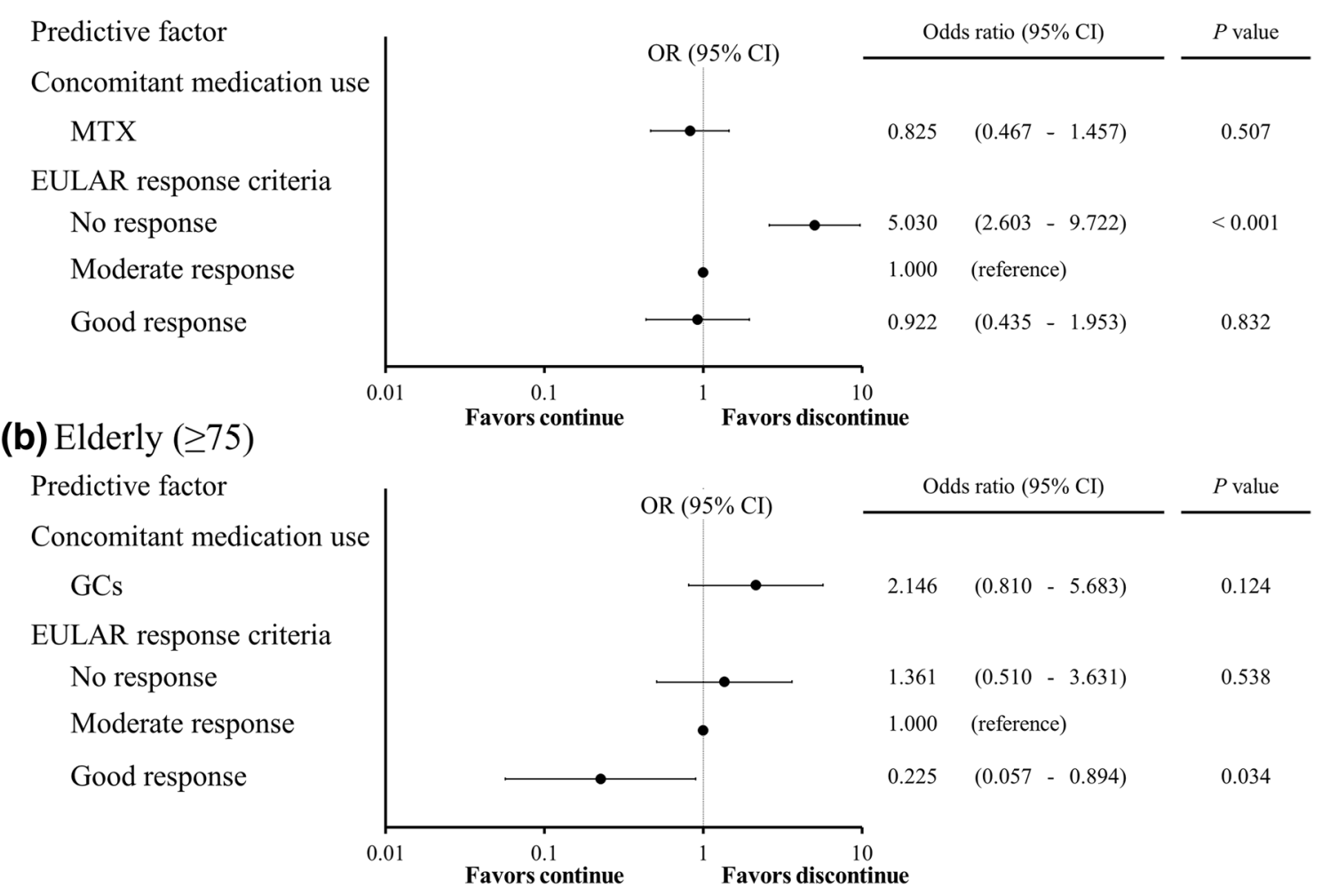

Fig. 2 Forest plots demonstrating patient factors associated with discontinuation of GLM treatment due to patient choice. Data are shown relative to EULAR moderate response. The vertical line represents no association with discontinuation of GLM treatment due to patient choice (odds ratio of 1.0). An odds ratio greater than $1.0(>1.0)$ favors discontinuation, whereas an odds ratio smaller than $1.0(<1.0)$ favors continuation. When

interest to more precisely pinpoint the factors associated with the discontinuation of GLM treatment in elderly RA patients. "Patient choice" in the current study is defined as a nonmedical decision made by patients independent of physicians' judgement. Since more elderly patients discontinued treatment than younger patients during the initial 4 weeks after starting GLM, one possibility might be that elderly patients were less responsive to GLM than younger patients due to their higher disease activities at baseline [44], urging them to prematurely judge that the treatment was not effective upon first administration and discouraging them from continuing GLM. In the confidence interval (CI) does not cross the vertical line, there is a statistically significant association between the factor and discontinuation of GLM treatment due to patient choice. EULAR response was evaluated following the final treatment including cases where patients discontinued GLM treatment before the completion of the 24-week study period

addition, when they have just started a biologic agent, physicians would emphasize potential occurrence of AEs and tell them to remain vigilant, possibly causing them to discontinue treatment due to anxiety about AEs. In fact, several studies reported that patients with rheumatic diseases share the beliefs that their medications are not helping or AEs are fearful, which are associated with discontinuation of treatment without a physician's recommendation $[45,46]$. These issues can be particularly problematic for elderly people because their ability to acquire and understand health information is markedly lower than younger people's [47]. 
Hence, to achieve sustained GLM treatment and the resultant clinical benefit for elderly RA patients, it would be of extreme importance for physicians to thoroughly communicate with elderly patients to help them understand their long-term goals together with the benefits and risks of GLM therapy. In fact, shared decisionmaking, as part of a successful treat-to-target approach [10,42], has been reported to improve patient satisfaction and adherence to medication in autoimmune diseases, allowing patients to achieve optimal management of their condition [48-50]. In addition, effective communication between a treating physician and a patient will enable the physician to promptly identify any potential AEs, ultimately leading to desirable clinical outcomes.

It is crucial to point out that our study is not only limited by its post hoc nature but also subject to notable limitations. First, the followup period of this study was short. Longer followup data collection would be necessary to determine any trends in cancer types in this patient population, although it is sufficient for the identification of the most serious infections [51]. Second, most analyses were performed within relatively small subgroups based on the original PMS data and thus random bias might not have been ruled out. Therefore, caution should be exercised in the interpretation and generalization of the factors found to be associated with continuation or discontinuation of GLM treatment. Third, since rheumatoid disease is commonly considered to be associated with a number of comorbidities and treatment with pharmacological drugs further complicates the contribution of the drug distinct from the disease effects, results need to be interpreted with caution. Forth, although there was no statistical difference in the overall incidences of AEs between younger and elderly patients, the interpretation of this analysis is limited by the fact that the AE types described in this study were broad and there were no specific AEs categorized according to the standardized system such as the Medical Dictionary for Regulatory Activities (MedDRA). Lastly, no causal relationship between the clinical outcomes and the patient characteristics can be derived from our study, thus requiring further studies to clarify such associations.

In summary, this post hoc study was one of the few that addressed the safety and effectiveness of biologics in the elderly population aged 75 years or older. While the strict eligibility criteria of clinical trials limited the generalizability of the efficacy and safety results in the elderly patients, our data here appear to be representative of a large retrospective clinical trial in Japan, suggesting that the safety and effectiveness of GLM treatment to patients with active RA aged 75 years or older are comparable to those in younger patients. In addition, within the limitations of the current study, our findings indicate that elderly patients are likely to discontinue GLM during early treatment phase by their own decision even before the full effect of GLM is expected. Therefore, continuous treatment with GLM for longer period might be a potential key to maximize the clinical benefit of GLM among elderly patients. Although we found that there is no significant difference in the effectiveness of GLM between the two age groups, further multicenter clinical studies of longer duration will be required to make a definitive recommendations and conclusions regarding the use of GLM to treat elderly RA patients in real-world clinical practice.

\section{ACKNOWLEDGEMENTS}

Funding. Sponsorship for this study and article processing charges were jointly provided by Janssen Pharmaceutical K.K. (Tokyo, Japan) and Mitsubishi Tanabe Pharma Corporation (Osaka, Japan). All authors had full access to all of the data in this study and take complete responsibility for the integrity of the data and accuracy of the data analysis.

Authorship. All named authors meet the International Committee of Medical Journal Editors (ICMJE) criteria for authorship for this article, take responsibility for the integrity of the work as a whole, and have given their approval for this version to be published. 
Medical Writing and/or Editorial Assistance. The authors would like to deeply acknowledge all patients and physicians who participated in the PMS study for their dedication. The authors also wish to thank Kenichirou Watanabe, Toshiro Yano, Toyokazu Saiki, Shigeru Matsuura, Fumikazu Okumura, and Takahiro Esaki (Ikuyaku. Integrated Value Development Division, Mitsubishi Tanabe Pharma Corporation) for their contribution to study design and data interpretation. Support for third-party writing assistance was provided by Dr. Negar Jamshid and funded by Janssen Pharmaceutical K.K. and Mitsubishi Tanabe Pharma Corporation.

Disclosures. Masateru Okazaki is an employee of Janssen Pharmaceutical K.K. and may hold stock and/or stock options in the company. Hisanori Kobayashi is an employee of Janssen Pharmaceutical K.K. and may hold stock and/or stock options in the company. Hirohito Shimizu is an employee of Janssen Pharmaceutical K.K. and may hold stock and/or stock options in the company. Yutaka Ishii is an employee of Janssen Pharmaceutical K.K. and may hold stock and/or stock options in the company. Tsutomu Yajima is an employee of Janssen Pharmaceutical K.K. and may hold stock and/or stock options in the company. Masayoshi Kanbori is an employee of Janssen Pharmaceutical K.K. and may hold stock and/or stock options in the company.

Compliance with Ethics Guidelines. This article is based on previously conducted postmarketing surveillance study and does not contain any new interventional studies with human participants or animal subjects performed by any of the authors. The PMS study protocol and ethical considerations were assessed by the internal review board members and approved by the Japanese PMDA. The PMS was conducted under the Japanese regulations (Ministry of Health, Labor and Welfare Ministerial Ordinance No. 171) for Good Post-Marketing Study Practice (GPSP), and was initiated after a contract with each study center.
Data Availability. The datasets generated during and/or analyzed during the current study are not publicly available due to confidentiality clauses signed with participating medical institutions.

Open Access. This article is distributed under the terms of the Creative Commons Attribution-NonCommercial 4.0 International License (http://creativecommons.org/licenses/ by-nc/4.0/), which permits any noncommercial use, distribution, and reproduction in any medium, provided you give appropriate credit to the original author(s) and the source, provide a link to the Creative Commons license, and indicate if changes were made.

\section{REFERENCES}

1. McInnes IB, Schett G. The pathogenesis of rheumatoid arthritis. $\mathrm{N}$ Engl J Med. 2011;365:2205-19.

2. Dougados M, Soubrier M, Antunez A, et al. Prevalence of comorbidities in rheumatoid arthritis and evaluation of their monitoring: results of an international, cross-sectional study (COMORA). Ann Rheum Dis. 2014;73:62-8.

3. Sparks JA, Chang SC, Liao KP, et al. Rheumatoid arthritis and mortality among women during 36 years of prospective follow-up: results from the Nurses' Health Study. Arthritis Care Res. 2016;68:753-62.

4. Gabriel SE. Cardiovascular morbidity and mortality in rheumatoid arthritis. Am J Med. 2008;121:S9-14.

5. Hunter TM, Boytsov NN, Zhang X, Schroeder K, Michaud K, Araujo AB. Prevalence of rheumatoid arthritis in the United States adult population in healthcare claims databases, 2004-2014. Rheumatol Int. 2017;37:1551-7.

6. Abhishek A, Doherty M, Kuo CF, Mallen CD, Zhang W, Grainge MJ. Rheumatoid arthritis is getting less frequent-results of a nationwide population-based cohort study. Rheumatology. 2017;56:736-44.

7. Yamanaka H, Sugiyama N, Inoue E, Taniguchi A, Momohara S. Estimates of the prevalence of and current treatment practices for rheumatoid arthritis in Japan using reimbursement data from health insurance societies and the IORRA cohort. Mod Rheumatol. 2014;24:33-40. 
8. Cross M, Smith E, Hoy D, et al. The global burden of rheumatoid arthritis: estimates from the Global Burden of Disease 2010 study. Ann Rheum Dis. 2014;73:1316-22.

9. Kato E, Sawada T, Tahara K, et al. The age at onset of rheumatoid arthritis is increasing in Japan: a nationwide database study. Int $J$ Rheum Dis. 2017;20:839-45.

10. Smolen JS, Breedveld FC, Burmester GR, et al. Treating rheumatoid arthritis to target: 2014 update of the recommendations of an international task force. Ann Rheum Dis. 2016;75:3-15.

11. Curtis JR, Singh JA. Use of biologics in rheumatoid arthritis: current and emerging paradigms of care. Clin Ther. 2011;33:679-707.

12. Chen J, Makovey J, Lassere M, Buchbinder R, March LM. Comparative effectiveness of anti-tumor necrosis factor drugs on health-related quality of life among patients with inflammatory arthritis. Arthritis Care Res. 2014;66:464-72.

13. Rossini M, De Vita S, Ferri C, et al. Golimumab: a novel anti-tumor necrosis factor. Biol Ther. 2013;3:83-107.

14. Zidi I, Bouaziz A, Mnif W, Bartegi A, Al-Hizab FA, Amor NB. Golimumab therapy of rheumatoid arthritis: an overview. Scand J Immunol. 2010;72:75-85.

15. Keystone EC, Genovese MC, Klareskog L, et al. Golimumab, a human antibody to TNF- $\alpha$ given by monthly subcutaneous injections, in active rheumatoid arthritis despite methotrexate: the GOFORWARD study. Ann Rheum Dis. 2009;68:789-96.

16. Inman RD, Davis JC, Heijde DV, et al. Efficacy and safety of golimumab in patients with ankylosing spondylitis: results of a randomized, double-blind, placebo-controlled, phase III trial. Arthritis Rheum. 2008;58:3402-12.

17. Kavanaugh A, McInnes I, Mease P, et al. Golimumab, a new human tumor necrosis factor $\alpha$ antibody, administered every four weeks as a subcutaneous injection in psoriatic arthritis: twentyfour-week efficacy and safety results of a randomized, placebo-controlled study. Arthritis Rheum. 2009;60:976-86.

18. Tanaka Y, Harigai M, Takeuchi T, et al. Golimumab in combination with methotrexate in Japanese patients with active rheumatoid arthritis: results of the GO-FORTH study. Ann Rheum Dis. 2012;71:817-24.

19. Takeuchi T, Harigai M, Tanaka Y, et al. Golimumab monotherapy in Japanese patients with active rheumatoid arthritis despite prior treatment with disease-modifying antirheumatic drugs: results of the phase $2 / 3$, multicentre, randomised, doubleblind, placebo-controlled GO-MONO study through 24 weeks. Ann Rheum Dis. 2013;72:1488-95.

20. Kanbori M, Suzuka H, Yajima T, et al. Postmarketing surveillance evaluating the safety and effectiveness of golimumab in Japanese patients with rheumatoid arthritis. Mod Rheumatol. 2018;28:66-75.

21. Wolfe F. The epidemiology of drug treatment failure in rheumatoid arthritis. Baillieres Clin Rheumatol. 1995;9:619-32.

22. van den Bemt BJ, Zwikker $\mathrm{HE}$, van den Ende $\mathrm{CH}$. Medication adherence in patients with rheumatoid arthritis: a critical appraisal of the existing literature. Expert Rev Clin Immunol. 2012;8:337-51.

23. Harrold LR, Andrade SE. Medication adherence of patients with selected rheumatic conditions: a systematic review of the literature. Semin Arthritis Rheum. 2009;38:396-402.

24. Cho SK, Sung YK, Kim D, et al. Drug retention and safety of TNF inhibitors in elderly patients with rheumatoid arthritis. BMC Musculoskelet Disord. 2016;17:333.

25. Mahlich J, Sruamsiri R. Persistence with biologic agents for the treatment of rheumatoid arthritis in Japan. Patient Prefer Adherence. 2016;10:1509-19.

26. Sakai R, Tanaka M, Nanki T, et al. Drug retention rates and relevant risk factors for drug discontinuation due to adverse events in rheumatoid arthritis patients receiving anticytokine therapy with different target molecules. Ann Rheum Dis. 2012;71:1820-6.

27. R Foundation for Statistical Computing. A language and environment for statistical computing. https:// www.R-project.org/. Accessed 1 Sept 2017.

28. Arai H, Ouchi Y, Toba K, et al. Japan as the frontrunner of super-aged societies: perspectives from medicine and medical care in Japan. Geriatr Gerontol Int. 2015;15:673-87.

29. Lahaye C, Tatar Z, Dubost JJ, Soubrier M. Overview of biologic treatments in the elderly. Jt Bone Spine. 2015;82:154-60.

30. Ouchi $\mathrm{Y}$, Rakugi $\mathrm{H}$, Arai $\mathrm{H}$, et al. Redefining the elderly as aged 75 years and older: proposal from the Joint Committee of Japan Gerontological Society and the Japan Geriatrics Society. Geriatr Gerontol Int. 2017;17:1045-7. 
31. Shono E. Effectiveness of golimumab in clinical management of patients with rheumatoid arthritis. Drugs R D. 2013;13:95-100.

32. Tanaka Y, Harigai M, Takeuchi T, et al. Clinical efficacy, radiographic progression, and safety through 156 weeks of therapy with subcutaneous golimumab in combination with methotrexate in Japanese patients with active rheumatoid arthritis despite prior methotrexate therapy: final results of the randomized GO-FORTH trial. Mod Rheumatol. 2016;26:481-90.

33. Japan College of Rheumatology 2016 guidelines for the use of methotrexate (MTX) in rheumatoid arthritis. Japan College of Rheumatology Subcommittee on Development of guidelines for the use of methotrexate in the treatment of rheumatoid arthritis. http://www.ryumachi-jp.com/publication/ pdf/MTX2016kanni.pdf. Accessed 8 Mar 2017.

34. Bathon JM, Fleischmann RM, Van der Heijde D, et al. Safety and efficacy of etanercept treatment in elderly subjects with rheumatoid arthritis. J Rheumatol. 2006;33:234-43.

35. Mueller RB, Kaegi T, Finckh A, Haile SR, SchulzeKoops $\mathrm{H}$, von Kempis J. Is radiographic progression of late-onset rheumatoid arthritis different from young-onset rheumatoid arthritis? Results from the Swiss prospective observational cohort. Rheumatology. 2013;53:671-7.

36. Yathish GC, Balakrishnan C, Mangat G, Parikshit S. Immunomodulators in managing geriatric rheumatoid arthritis. Int J Clin Rheumatol. 2015;3:SR2.

37. Morović-Vergles J, Salamon L, Marasović-Krstulović $\mathrm{D}$, et al. Is the prevalence of arterial hypertension in rheumatoid arthritis and osteoarthritis associated with disease? Rheumatol Int. 2013;33:1185-92.

38. Burner TW, Rosenthal AK. Diabetes and rheumatic diseases. Curr Opin Rheumatol. 2009;21:50-4.

39. Radner H, Lesperance T, Accortt NA, Solomon DH. Incidence and prevalence of cardiovascular risk factors among patients with rheumatoid arthritis, psoriasis, or psoriatic arthritis. Arthritis Care Res. 2017;69:1510-8.

40. Maradit-Kremers H, Nicola PJ, Crowson CS, Ballman KV, Gabriel SE. Cardiovascular death in rheumatoid arthritis: a population-based study. Arthritis Rheum. 2005;52:722-32.

41. Furst DE, Kremer JM. Methotrexate in rheumatoid arthritis. Arthritis Rheum. 1988;31:305-14.
42. Smolen JS, Landewé R, Breedveld FC, et al. EULAR recommendations for the management of rheumatoid arthritis with synthetic and biological disease-modifying antirheumatic drugs: 2013 update. Ann Rheum Dis. 2014;73:492-509.

43. Barbosa CD, Balp MM, Kulich K, Germain N, Rofail D. A literature review to explore the link between treatment satisfaction and adherence, compliance, and persistence. Patient Prefer Adherence. 2012;6:39-48.

44. Aletaha D, Funovits J, Keystone EC, Smolen JS. Disease activity early in the course of treatment predicts response to therapy after one year in rheumatoid arthritis patients. Arthritis Rheum. 2007;56:3226-35.

45. Garcia Popa-Lisseanu MG, Greisinger A, Richardson $\mathrm{M}$, et al. Determinants of treatment adherence in ethnically diverse, economically disadvantaged patients with rheumatic disease. J Rheumatol. 2005;32:913-9.

46. Linde L, Hetland ML, Ãstergaard M. Drug survival and reasons for discontinuation of intramuscular methotrexate: a study of 212 consecutive patients switching from oral methotrexate. Scand J Rheumatol. 2006;35:102-6.

47. Baker DW, Gazmararian JA, Sudano J, Patterson M. The association between age and health literacy among elderly persons. J Gerontol B Psychol Sci Soc Sci. 2000;55:S368-74.

48. Barton JL. Patient preferences and satisfaction in the treatment of rheumatoid arthritis with biologic therapy. Patient Prefer Adherence. 2009;3:335-44.

49. Chilton F, Collett RA. Treatment choices, preferences and decision-making by patients with rheumatoid arthritis. Musculoskeletal Care. 2008;6:1-4.

50. Lofland JH, Johnson PT, Ingham MP, Rosemas SC, White JC, Ellis L. Shared decision-making for biologic treatment of autoimmune disease: influence on adherence, persistence, satisfaction, and health care costs. Patient Prefer Adherence. 2017;11:947-58.

51. Emery P, Fleischmann RM, Moreland LW, et al. Golimumab, a human anti-tumor necrosis factor alpha monoclonal antibody, injected subcutaneously every four weeks in methotrexate-naive patients with active rheumatoid arthritis: twentyfour-week results of a phase III, multicenter, randomized, double-blind, placebo-controlled study of golimumab before methotrexate as first-line therapy for early-onset rheumatoid arthritis. Arthritis Rheum. 2009;60:2272-83. 